\title{
Positron identification in the ENUBET instrumented decay tunnel
}

\section{Fabio Pupilli*}

INFN - Sezione di Padova

E-mail: fabio.pupilliepd.infn.it

G. Ballerini ${ }^{a, b}$, A. Berra ${ }^{a, b}$, R. Boanta ${ }^{b, h}$, M. Bonesini ${ }^{b}$, C. Brizzolari ${ }^{a, b}$, G. Brunetti ${ }^{j}$, M. G. Catanesi $i^{l}$, S. Cecchini ${ }^{c}$, F. Cindolo ${ }^{c}$, A. Coffani ${ }^{b, h}$, G. Collazuol ${ }^{k, j}$, E. Conti ${ }^{j}$, F. Dal Corso ${ }^{j}$, G. De Rosa ${ }^{p, q}$, A. Gola ${ }^{o}$, R. A. Intonti ${ }^{l}$, C. Jollet ${ }^{d}$, Y. Kudenko ${ }^{r}$, M. Laveder ${ }^{k, j}$, A. Longhin ${ }^{j}$, P.F. Loverre ${ }^{n, f}$, L. Ludovici ${ }^{f}$, L. Magaletti ${ }^{l}$, G. Mandrioli ${ }^{c}$, A. Margotti ${ }^{c}$, V. Mascagna ${ }^{a, b}$, N. Mauri ${ }^{c}$, A. Meregaglia ${ }^{s}$, M. Mezzetto $^{j}$, M. Nessi ${ }^{m}$, A. Paoloni ${ }^{e}$, M. Pari ${ }^{k, j}$, G. Paternoster ${ }^{o}$, L. Patrizii ${ }^{c}$, C. Piemonte ${ }^{o}$, M. Pozzato ${ }^{c}$, M. Prest ${ }^{a, b}$, E. Radicioni ${ }^{l}$, C. Riccio ${ }^{p, q}$, A. C. Ruggieri ${ }^{p}$, M. Soldani ${ }^{a, b}$, G. Sirri ${ }^{c}$, M. Tenti ${ }^{c, g}$, F. Terranova ${ }^{a, b}$, E. Vallazza ${ }^{i}$, L. Votano $^{e}$, E. Wildner ${ }^{m}$

${ }^{a}$ Phys. Dep., Università degli studi dell'Insubria, via Valeggio 11, Como

${ }^{b}$ INFN, Sezione di Milano-Bicocca, piazza della Scienza 3, Milano, Italy

${ }^{c}$ INFN, Sezione di Bologna, viale Berti-Pichat 6/2, Bologna, Italy

${ }^{d}$ IPHC, Université de Strasbourg, CNRS/IN2P3, Strasbourg, France

e INFN, Laboratori Nazionali di Frascati, via Fermi 40, Frascati (Rome), Italy

${ }^{f}$ INFN, Sezione di Roma 1, piazzale A. Moro 2, Rome, Italy

${ }^{g}$ Phys. Dep. Università di Bologna, viale Berti-Pichat 6/2, Bologna, Italy

${ }^{h}$ Phys. Dep. Università di Milano-Bicocca, piazza della Scienza 3, Milano, Italy

${ }^{i}$ INFN Sezione di Trieste, via Valerio, 2 - Trieste, Italy

${ }^{j}$ INFN Sezione di Padova, via Marzolo, 8 - Padova, Italy

${ }^{k}$ Phys. Dep. Università di Padova, via Marzolo, 8 - Padova, Italy

${ }^{l}$ INFN Sezione di Bari, via Amendola, 173 - Bari, Italy

${ }^{m}$ CERN, Geneva, Switzerland

${ }^{n}$ Phys. Dep. Università La Sapienza, piazzale A. Moro 2, Rome, Italy

${ }^{o}$ Fondazione Bruno Kessler (FBK) and INFN TIFPA, Trento, Italy

${ }^{p}$ INFN, Sezione di Napoli, via Cinthia, 80126, Napoli, Italy

${ }^{q}$ Phys. Dep. Università degli Studi di Napoli Federico II, via Cinthia, 80126, Napoli

${ }^{r}$ Institute of Nuclear Research of the Russian Academy of Science, Moscow, Russia

${ }^{s}$ CENBG, Université de Bordeaux, CNRS/IN2P3, 33175 Gradignan, France 
The ERC granted ENUBET project aims at developing the technologies to reduce by a factor $\sim 10$ the systematics in neutrino fluxes from conventional beams, allowing measuring the $v_{e}$ (and $\bar{v}_{e}$ ) cross section with a $1 \%$ precision, in the region of interest for future oscillation experiments looking for CP violation. This goal is accomplished by monitoring in an instrumented decay tunnel the high angle positron produced in $\mathrm{K}_{e 3}$ decays of charged kaons. The Monte Carlo simulation of the positron tagger in realistic conditions and a preliminary event reconstruction chain will be described, together with results on the expected signal selection efficiency.

XVII International Workshop on Neutrino Telescopes

13-17 March 2017

Venezia, Italy

${ }^{*}$ Speaker. 


\section{ENUBET}

The main limiting factor for a precise measurement of neutrino cross sections is represented by systematic uncertainties in the assessment of the initial flux, that is performed through a complete simulation of the production beamline. Despite the use of hadro-production data from dedicated experiments and of ancillary measurements (proton intensity, horn currents, muon monitoring after the beam dump), the precision on the flux prediction is usually limited to 7-10\%. This uncertainty also affects the $v_{e}$ cross section, which is poorly known in spite of its relevance for precision oscillation physics in the next generation of long-baseline experiments. The direct monitoring of leptons produced in the decay tunnel with the neutrinos can overcome these limitations and significantly reduce the flux-related systematics. If the decay tunnel is short enough and the momentum of secondary hadrons is of the order of few $\mathrm{GeV}$, the only source of $v_{e}$ in the beamline is the three body decay of charged kaons $\left(K_{e 3}\right): k^{+} \rightarrow e^{+} \pi^{0} v_{e}$. Instrumenting the decay tunnel with fast calorimetric modules allows the tagging of the emitted large angle positrons and a direct estimation of the electron neutrino flux [1].

The ENUBET project is intended to demonstrate the feasibility of this approach; it has been funded by the European Research Council (ERC Consolidator Grant, PI A. Longhin, host institution INFN) for a five year duration, starting from June 2016. The reference design [2] foresees a secondary hadron beam of $8.5 \pm 20 \% \mathrm{GeV} / \mathrm{c}$ momentum. Its emittance and the radius of the $50 \mathrm{~m}$ decay tunnel are optimized in order to prevent muons from pions or undecayed secondaries from hitting the instrumented walls of the tunnel. The calorimetric solution for the tunnel instrumentation allows for a cost-effective and efficient separation of $K_{e 3}$ positrons from muons and pions produced in other kaon decay modes. It is based on units called Ultra Compact Modules (UCM) made of five, $15 \mathrm{~mm}$ thick, iron layers, interleaved by $5 \mathrm{~mm}$ thick plastic scintillator tiles, that instruments the two inner layers of the calorimeter. The total length of the module $(10 \mathrm{~cm})$ corresponds to $4.3 X_{0}$ and its transverse size is of $3 \times 3 \mathrm{~cm}^{2}$. The readout is performed in shashlik mode through nine WLS fibers coupled to silicon photomultipliers. Six outer layers of shashlik modules with a longitudinal coarser readout (every $60 \mathrm{~cm}$, i.e. 2.6 interaction lengths) complete the calorimeter and are used to measure the energy released by pions and muons, while e.m. showers will release their energy in the two innermost ones. Rings of two plastic scintillator pads with a $3 \times 3 \mathrm{~cm}^{2}$ surface are displaced below the calorimeter at every $7 \mathrm{~cm}$ and are used to suppress the photon background from $\pi^{0}$ decays and to provide the reference time of an event (" $\mathrm{t}_{0}$ layer"). Further details on the full ENUBET setup, its physics potential, the prototyping activities and the achievements of the first year of the project can be found in [3].

\section{Simulation of the instrumented decay tunnel and event reconstruction}

A full GEANT4 [4] simulation of the instrumented tunnel has been setup in order to test his performances in terms of particle identification and to optimize his design. Secondary mesons are generated at the tunnel entrance with emittance and momentum distributions defined in the reference design. More realistic distributions will be implemented as soon as the ongoing optimization of the focusing and transfer line will be completed. 
A preliminary event reconstruction chain has been defined [5]. The preliminary step is the event building to correlate in space and time energy deposits in different UCM along the tunnel belonging to the same decay, and to deal with the pile-up induced by other decays; indeed, with the meson flux assumed in [1] (about $5 \times 10^{11} \mathrm{~K}^{+}$per second), a particle rate of $500 \mathrm{kHz} / \mathrm{cm}^{2}$ is estimated at the inner surface of the calorimeter. For each spill, the first UCM in time in the innermost layer with a visible energy exceeding $20 \mathrm{MeV}$ is selected as "seed" for the event reconstruction, thus enhancing the preselection of positron events. UCM and $t_{0}$ signals correlated to the seed are clustered taking into account their position and timing. The procedure is iterated over all the signals recorded.

A multivariate analysis based on a neural network is then applied to the reconstructed events for the discrimination between charged pions and positrons; it relies on the pattern of energy deposit in the calorimeter through five variables:

- the ratio among the most energetic signal in the first electromagnetic layer and the total visible energy in all layers;

- the fraction of energy released in the first layer;

- the fraction of energy released in the first and second layers;

- the fraction of energy released in the two adjacent longitudinal rows in the transverse plane with respect to the one of the seed;

- the total visible energy.

The response of the neural network to pure positron and pion samples is shown in Fig. 1.

\section{TMVA overtraining check for classifier: MLPBNN}

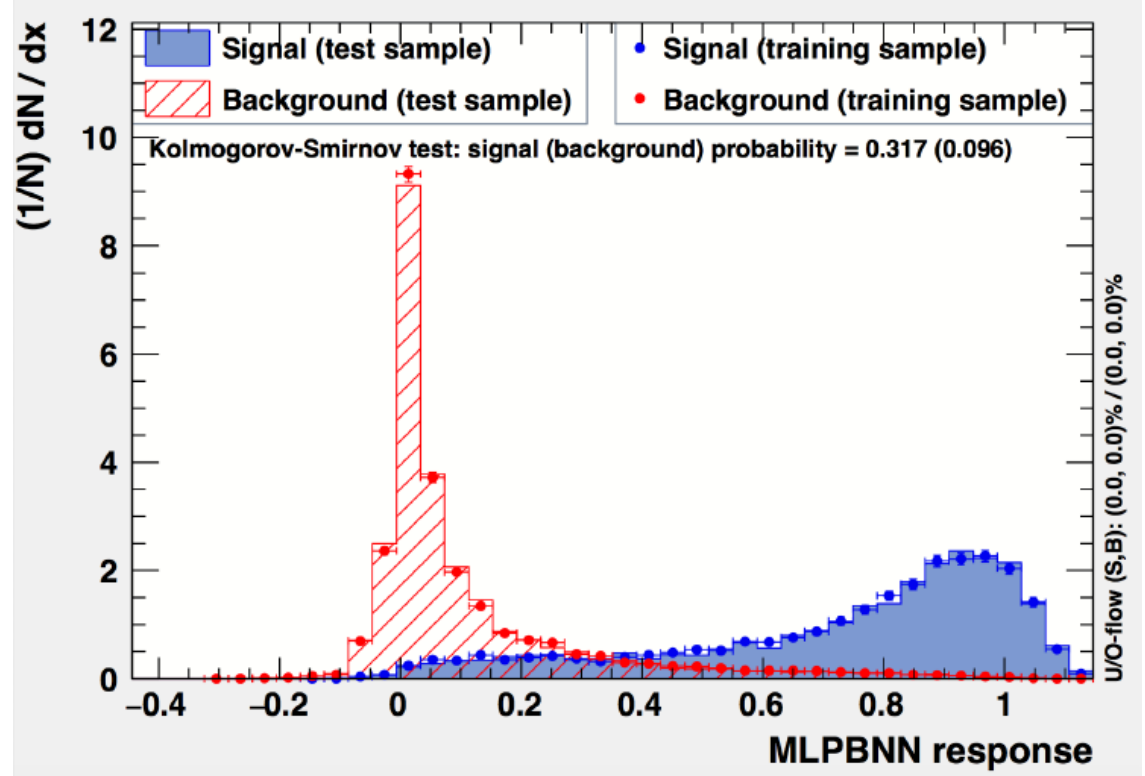

Figure 1: TMVA [6] neural network response for $\pi^{ \pm}$(red) and $e^{+}$(blue). 
As a final step, sequential cuts based on the informations from the photon veto are applied to reject $\pi_{0}$-related events: it is required that the three $t_{0}$ pads before the seed longitudinal position measure an energy in the $0.65-1.7 \mathrm{MeV}$ range, compatible with the energy deposit of just one minimum ionizing particle (thus also suppressing $\gamma$ conversions in the scintillator).

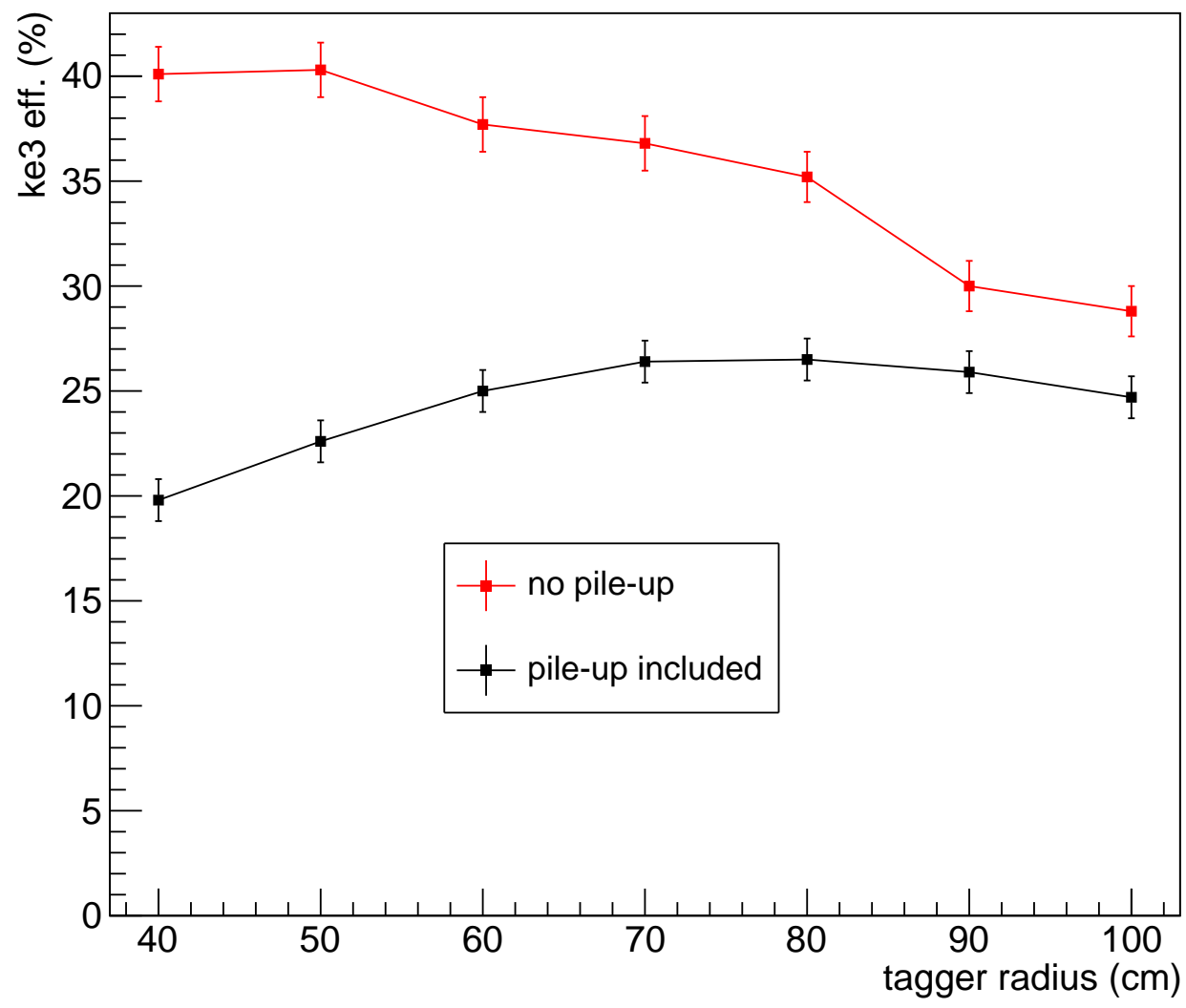

Figure 2: Ke3 selection efficiency as a function of the decay tunnel radius. In red are shown efficiencies when events are temporally separated. In black are reported the efficiencies when including pile-up effects at the nominal meson rate.

As can be seen in Fig. 2, the $K_{e 3}$ selection efficiency, that amounts to $\sim 40 \%$ in ideal conditions and with a tunnel radius of $40 \mathrm{~cm}$, is degraded by pile-up effects to below $20 \%$. A mitigation can be obtained by enlarging the tunnel at the expanse of a reduced geometrical acceptance, with a final tagging efficiency of about $25 \%$ putting the calorimeter at $1 \mathrm{~m}$ from the beam axis. In these conditions the purity of the selection amounts to $\sim 50 \%$ and Fig. 3 shows the contributions from mis-identified events. A larger tunnel radius is also beneficial in terms of less stringent emittance requirements for the focusing line and for the radiation hardness properties of the instrumentation [3].

Additional studies are ongoing to further optimize the tagger, testing different calorimetric module granularities and the possibility of reducing the number of outer layers and/or the instrumented fraction of the tunnel, with the final goal to asses the systematics to the neutrino flux due 


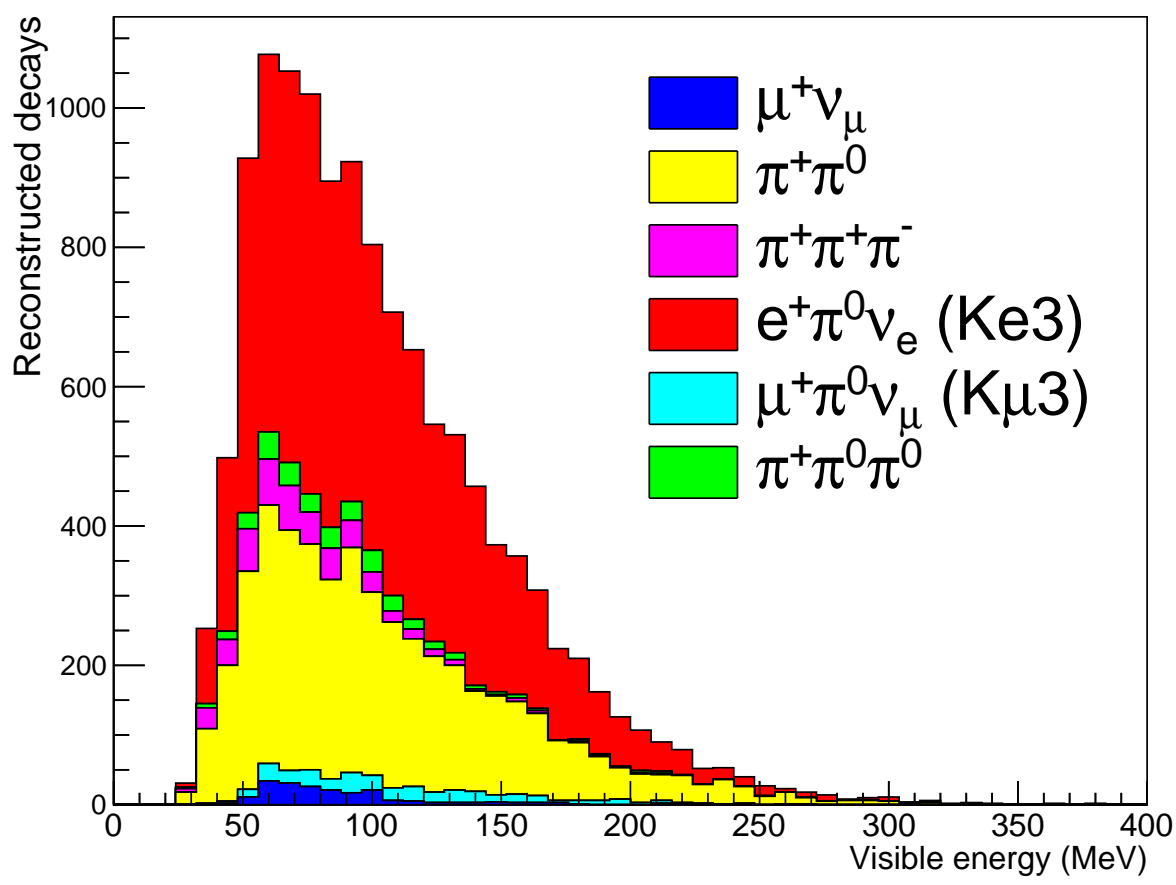

Figure 3: Contribution of the different kaon decay modes to all the events reconstructed as $K_{e 3}$

to the detector response.

\section{Acknowledgements}

This project has received funding from the European Union's Horizon 2020 Research and Innovation programme under Grant Agreement no. 654168 and no. 681647.

\section{References}

[1] A. Longhin, L. Ludovici and F. Terranova, Eur. Phys. J. C 75, 155 (2015).

[2] A. Berra et al. [ENUBET Coll.], CERN-SPSC-2016-036; SPSC-EOI-014.

[3] A. Longhin et al., these proceedings.

[4] S. Agostinelli et al., [GEANT4 Coll.], Nucl. Instrum. Meth. A 506, 250 (2003).

[5] A. Meregaglia et al., JINST 11 no.12, C12040 (2016).

[6] A. Hoecker et al., PoS ACAT, 040 (2007). 